\title{
Generalized Point Estimators for Fuzzy Multivariate Data
}

\author{
Owat Sunanta \\ Vienna University of Technology
}

\begin{abstract}
Data analysis methods are necessary tools in evaluating and for better understanding the information of interest. However, there are limitations in applying standard statistical methods to data analysis in some cases. Data obtained from different sources are often clouded by imprecision and uncertainty, also called fuzziness. To overcome this problem, data analysis has to be adapted and generalized through statistical methods for such fuzzy data to capture the uncertainty. These methods are largely based on the extension principle and/or require other generalized procedures for further calculation of statistics, e.g. the estimation of the unknown statistical parameters. The development of these methods specifically for evaluating univariate data has been flourished. However, to solve complex real-world problems, these methods have to be extended and generalized to handle multivariate fuzzy data. In this research, the methods of generalized point estimators, i.e. sample mean, variance-covariance, and correlation coefficient, are extended for the multivariate case through concepts of fuzzy vector and combined fuzzy sample.
\end{abstract}

Keywords: fuzzy multivariate data, fuzzy vector, combined fuzzy sample, multivariate statistical analysis.

\section{Introduction}

Multivariate statistical analysis is concerned with data frequently obtained from empirical measurements on a number of individuals or objects. The sample data or the statistical treatments may be made on a collection of measurements in a manufacturing environment, such as diameter and surface finish of valve seats and those of corresponding nozzle seats in a petroleum system. The measurements made on a single unit (or a specimen) can ideally be assembled into a column vector. The entire vector may be thought of as an observation from a multivariate population or distribution. In statistical terms, when the individual measure is drawn randomly, the resulting vector is considered a random vector with a distribution or probability law describing the population (Anderson 2003). The set of observations on all individuals in a sample constitutes a sample of vectors, and the vectors set side by side make up the matrix of observations. The data to be analyzed are then thought of as displays in a matrix or in several matrices.

Unfortunately, the measurements are often clouded especially by uncertainty and imprecision. The uncertainty of measurement results of continuous quantities differs from probabilistic un- 
certainty, however. Individual measurement results also contain a kind of uncertainty, which is called fuzziness. The most suitable mathematical model to describe the fuzziness is by fuzzy numbers and their characterizing functions (Zadeh 1965), (Viertl 2015). The extension principle (Zadeh 1975) in fuzzy set theory has been used in applications which call for an extension of the domain of a relation. In terms of statistical inference for fuzzy data, some literatures present formulating the statistics as by-product of fuzzy random variables Kruse (1984), Kruse and Meyer (1987), Liu and Kao (2002), Nguyen and Wu (2006), Wang (2004). In most cases, the notion of expected values is defined for fuzzy numbers of intervals as in (Dubois and Prade 1987), viewed as random fuzzy sets, which are subsequently used in analyzing fuzzy data. On the other hand, descriptive statistics through point estimators can be formulated differently through embedding the fuzziness through mathematical structures, a focal point of this paper. For univariate data, statistical inference involves combining fuzzy samples and propagation of fuzziness through fuzzy mathematical functions as reported by (Frühwirth-Schnatter 1992), (Viertl 2006), (Viertl 2011).

For analysis of multivariate fuzzy data, displayed in form of matrices of fuzzy numbers, generalized standard statistical inference methods for multivariate fuzzy data have to be considered. Comprehensive literatures in this particular area are limited and/or the proposed definitions are not necessarily concise. In case of multivariate fuzzy data, statistical inferences via point estimators require mathematical statements and involve high-dimension Cartesian products as a result of two-way combining of samples and multiple variables. With combined samples, further succinct mathematical definitions are possible. These concepts will be explained in detail and soon become obvious to the readers in the subsequent sections. For variance/covariance matrices, one of the differences in definitions lies in how the quantification methods of distances between the observed values (samples) and their respective mean are defined, see (Blanco-Fernàndez et al. 2014), (Körner 1997), (Kruse 1987), (Voxman 1998), and (Wang 2004) for different definitions, which serve different purposes, i.e. not necessary a generalized method for an $\mathbb{R}^{k \cdot n}$ space. Aside from variance, the measure of association through estimators of the population correlation coefficient is often of interest. In general, for fuzzy data, the correlation coefficient obtained through the Extension Principle is fuzzy as well (Ni and Cheung 2003). Different definitions of fuzzy correlation coefficient have been introduced, some of which result in the possible values of the correlation coefficient within $[0,1]$ (Bustince and Burillo 1995) as opposed to within [-1,1] as defined in (Saneifard and Saneifard 2012) and (Liu and Kao 2002). The latter is the same definition as in standard case and also adopted here.

This paper presents a statistical inference method through mathematical statements and generalized point estimators for fuzzy multivariate data. Section 2 provides the foundation of fuzzy numbers and fuzzy vectors along with a concept of combining fuzzy samples in univariate

case. In section 3 , fuzzy multivariate data are described. In section 4 , statistical functions of fuzzy multivariate data are then presented. The foundations presented in the previous sections are then applied in generalizing the point estimators along with a numerical example as shown in section 5. The paper concludes with final remarks.

\section{Fuzzy numbers, fuzzy vectors and combined fuzzy samples}

In case of univariate data, to describe observations or measurements of continuous quantities, the definition of general fuzzy numbers is useful.

Definition 1. A general fuzzy number $x^{\star}$ is defined by its characterizing function $\xi(\cdot)$, which is a real function of one real variable and possesses the following properties:

(1) $\xi: \mathbb{R} \rightarrow[0,1]$

(2) The support of $\xi(\cdot)$, denoted by $\operatorname{supp}[\xi(\cdot)]$ and defined by $\operatorname{supp}[\xi(\cdot)]:=\{x \in \mathbb{R}: \xi(x)>0\}$, is a bounded subset of $\mathbb{R}$. 
(3) For all $\delta \in(0,1]$, the $\delta$-cut $C_{\delta}[\xi(\cdot)]$, defined by $C_{\delta}[\xi(\cdot)]:=\{x \in \mathbb{R}: \xi(x) \geq \delta\}=\bigcup_{j=1}^{n_{\delta}}\left[a_{\delta, j} ; b_{\delta, j}\right]$, is non-empty and a finite union of compact intervals.

Along with general fuzzy numbers, how to obtain the characterizing function of a measurement result is also critical. For example, if a measurement $x^{\star}$ is quantified and represented by an interval $[a, b]$, such an interval can be characterized by its indicator function $\mathbb{1}_{[a, b]}(\cdot)$, i.e. $\xi(\cdot)$ $=\mathbb{1}_{[a, b]}(\cdot)$, which is, in fact, a special kind of characterizing function. On the other hand, if a measurement $x^{\star}$ is recognized by a function $h(\cdot)$, the characterizing function of $x^{\star}$ can be derived through a transformation of its inherent function $h(\cdot)$, as following:

$$
\xi(x):=\frac{h(x)}{\max \{h(x): x \in \mathbb{R}\}} \quad \forall x \in \mathbb{R} .
$$

Further details and examples can be found in Klir and Yuan (1995) and Viertl (2011).

Definition 2. Let $x_{1}^{\star}, \ldots, x_{n}^{\star}$ be $n$ fuzzy numbers of the observation space $M_{x} \subseteq \mathbb{R}$ with corresponding characterizing functions $\xi_{1}(\cdot), \ldots, \xi_{n}(\cdot)$. To obtain a fuzzy vector $\mathbf{x}^{\star}$, the fuzzy numbers $x_{1}^{\star}, \ldots, x_{n}^{\star}$ have to be combined (Viertl and Hareter 2006). Through construction of an $n$-dimensional vector-characterizing function $\zeta(\cdot, \ldots, \cdot)$ via a triangular norm $(t$-norm $T)$, the combined fuzzy sample $\mathbf{x}^{\star}$ forms a fuzzy element $\left(x_{1}, \ldots, x_{n}\right)^{\star}$ of the sample space $M_{x}^{n}$, i.e.

$x_{i}^{\star} \widehat{=} \xi_{i}(\cdot), i=1(1) n \stackrel{t-\text { norm } T}{\Longrightarrow}$ combined fuzzy sample $\mathbf{x}^{\star}$ and vector-characterizing function $\zeta(\cdot, \ldots, \cdot)$ where $\zeta\left(x_{1}, \ldots, x_{n}\right):=\kappa_{n}\left[\xi_{1}\left(x_{1}\right), \ldots, \xi_{n}\left(x_{n}\right)\right] \quad \forall\left(x_{1}, \ldots, x_{n}\right) \in \mathbb{R}^{n}$

and the combination $\kappa_{n}=T_{n}$, which is the $n$-dimensional extension of the $t$-norm $T$ by its associativity, i.e. $T_{n}\left(y_{1}, \ldots, y_{n}\right)=T\left(y_{1}, T\left(\ldots, T\left(y_{n-1}, y_{n}\right) \ldots\right)\right) \quad \forall\left(y_{1}, \ldots, y_{n}\right) \in[0,1]^{n}$.

For statistical and algebraic calculations with fuzzy data, the minimum- $t$-norm $T$ is optimal (Viertl 2011), i.e.

$$
\zeta\left(x_{1}, \ldots, x_{n}\right)=T_{n}\left(\xi_{1}\left(x_{1}\right), \ldots, \xi_{n}\left(x_{n}\right)\right)=\min \left\{\xi_{1}\left(x_{1}\right), \ldots, \xi_{n}\left(x_{n}\right)\right\} \quad \forall\left(x_{1}, \ldots, x_{n}\right) \in \mathbb{R}^{n} .
$$

Lemma 1. A fuzzy vector $\boldsymbol{x}^{\star}$ is obtained via minimum-t-norm when the individual values of the variables $x_{i}$ are fuzzy numbers $x_{i}^{\star}$. Through the minimum-t-norm, the combination of $n$ fuzzy numbers with characterizing functions $\xi_{i}(\cdot), i=1(1) n$, a fuzzy vector $\boldsymbol{x}^{\star}=\left(x_{1}, \ldots, x_{n}\right)^{\star}$ is obtained. In this case, the following holds:

$$
C_{\delta}[\zeta(\cdot, \ldots, \cdot)]=\mathrm{X}_{i=1}^{n} C_{\delta}\left[\xi_{i}(\cdot)\right] \quad \forall \delta \in(0,1]
$$

In words, the $\delta$-cuts of the fuzzy vector $\boldsymbol{x}^{\star}=\left(x_{1}, \ldots, x_{n}\right)^{\star}$ are the Cartesian products of the $\delta$-cuts of the fuzzy numbers $x_{i}^{\star}, i=1(1) n$.

$$
\text { Proof. } \quad \begin{aligned}
C_{\delta}[\zeta(\cdot, \ldots, \cdot)]= & \left\{\mathbf{x} \in \mathbb{R}^{n}: \zeta(\mathbf{x} \geq \delta\}\right. \\
= & \left\{\mathbf{x}: \min \left\{\xi_{1}\left(x_{1}\right), \ldots, \xi_{n}\left(x_{n}\right)\right\} \geq \delta\right\} \\
= & X_{i=1}^{n} C_{\delta}\left[\xi_{i}(\cdot)\right]
\end{aligned}
$$

The concepts of combined fuzzy samples and triangular norms are useful for succinct multivariate statistical analysis of fuzzy data.

\section{Fuzzy multivariate data}

For multivariate continuous data, i.e. one observation with $k$ variables (dimensions), an idealized measurement results in a $k$-dimensional real vector $\left(x_{1}, \ldots, x_{k}\right)$. In reality, there are two possibilities:

First, when the individual values of the variables $x_{i}$ are fuzzy numbers $x_{i}^{\star}$, a vector of fuzzy numbers $\left(x_{1}^{\star}, \ldots, x_{k}^{\star}\right)$ is obtained. In this case, it is necessary to combine the fuzzy 
numbers $x_{1}^{\star}, \ldots, x_{k}^{\star}$ to obtain a fuzzy element $\left(x_{1}, \ldots, x_{k}\right)^{\star}$ of the observation space $M_{x}^{k} \in \mathbb{R}^{k}$. This combination is accomplished by a suitable $t$-norm $T$ and its extension $T_{k}$ on $[0,1]^{k}$.

Second, when the vector itself is fuzzy, hence, a fuzzy version of a vector $\left(x_{1}, \ldots, x_{k}\right)^{\star}$, e.g. fuzzy point in $\mathbb{R}^{k}$, is obtained. For example, ideally, the position of a ship on a radar screen is a two-dimensional vector $\left(x_{1}, x_{2}\right)$ or $(x, y) \in \mathbb{R}^{2}$. In real situations, such position is characterized by a light point on the radar screen, which is not a precise vector, i.e. fuzzy vector.

Definition 3. Using the notation $\mathbf{x}=\left(x_{1}, \ldots, x_{k}\right)$, a $k$-dimensional fuzzy vector $\mathbf{x}^{\star}$ is determined by its so-called vector-characterizing function $\zeta(\cdot, \ldots, \cdot)$, which is a real function of $k$ real variables $x_{1}, \ldots, x_{k}$ and possesses the following properties (Viertl and Sunanta 2013):

(1) $\zeta: \mathbb{R}^{k} \rightarrow[0,1]$

(2) The support of $\zeta(\cdot, \ldots, \cdot)$ is a bounded set.

(3) For all $\delta \in(0,1]$, the $\delta$-cut $C_{\delta}\left[\mathbf{x}^{\star}\right]$, defined by

$C_{\delta}\left[\mathbf{x}^{\star}\right]:=\left\{\mathbf{x} \in \mathbb{R}^{k}: \zeta(\mathbf{x}) \geq \delta\right\}$

is non-empty and a finite union of simply connected and closed sets.

The mathematical formalization of the combined fuzzy samples and that of the fuzzy version of a vector are described here respectively.

If only fuzzy coordinates $x_{j}^{\star}, j=1(1) k$ are available, in order to obtain the vector-characterizing function of the generated fuzzy vector, the characterizing functions of the fuzzy coordinates $x_{j}^{\star}$ have to be combined. In this case, the vector-characterizing function of a fuzzy version of a vector $\left(x_{1}, \ldots, x_{k}\right)^{\star}$ can be derived through the product- $t$-norm:

$$
\zeta\left(x_{1}, \ldots, x_{n}\right) \quad:=\prod_{j=1}^{k} \xi_{j}\left(x_{j}\right) \quad \forall\left(x_{1}, \ldots, x_{k}\right) \in \mathbb{R}^{k}
$$

For the special case $k=2$, that is when fuzzy coordinates $x_{1}^{\star}$ and $x_{2}^{\star}$ of a vector, with characterizing functions $\xi_{1}(\cdot)$ and $\xi_{2}(\cdot)$ respectively, are measured, the vector-characterizing function $\zeta(\cdot, \cdot)$ of the fuzzy vector $\mathbf{x}^{\star}=\left(x_{1}, x_{2}\right)^{\star}$ is obtained via the product- $t$-norm $T_{\text {prod }}$, i.e.

$$
\zeta\left(x_{1}, x_{2}\right)=T_{\text {prod }}\left(\xi_{1}\left(x_{1}\right), \xi_{2}\left(x_{2}\right)\right)=\xi_{1}\left(x_{1}\right) \cdot \xi_{2}\left(x_{2}\right) \quad \forall\left(x_{1}, x_{2}\right) \in \mathbb{R}^{2}
$$

The result is a fuzzy vector, denoted as $\left(x_{1}, \ldots, x_{k}\right)^{\star}$ with a vector-characterizing function $\zeta\left(x_{1}, x_{2}\right)=\xi_{1}\left(x_{1}\right) \cdot \xi_{2}\left(x_{2}\right) \quad \forall\left(x_{1}, x_{2}\right) \in \mathbb{R}^{2}$ where $\xi_{1}(\cdot)$ and $\xi_{2}(\cdot)$ are the characterizing functions of the fuzzy coordinates $x_{1}^{\star}$ and $x_{2}^{\star}$ respectively.

In case of a sample of fuzzy observations $\mathbf{x}_{i}^{\star}, i=1(1) n$, with $k$-dimensional vector-characterizing functions $\zeta_{i}\left(x_{1}, \ldots, x_{k}\right)$, the generalized minimum rule is applied in oder to obtain the vectorcharacterizing function $\zeta\left(x_{1}, \ldots, x_{k \cdot n}\right)$ for the combined fuzzy vector $\mathbf{X}^{\star}$, which is the combined fuzzy sample. Considering a fuzzy sample $\mathbf{x}_{1}^{\star}, \ldots, \mathbf{x}_{n}^{\star}$ with its corresponding vectorcharacterizing functions $\zeta_{i}(\cdot, \ldots, \cdot)$, where $\mathbf{x}=\left(x_{1}, \ldots, x_{k}\right) \in \mathbb{R}^{k}$ and sample space $\mathbb{R}^{k \cdot n}$, the followings can be defined:

Let $\mathbf{x}_{i}^{\star} \in \mathcal{F}\left(\mathbb{R}^{k}\right), i=1(1) n$ and the combined fuzzy sample $\mathbf{X}^{\star} \in \mathcal{F}\left(\mathbb{R}^{k \cdot n}\right)$, the vectorcharacterizing function $\zeta(\cdot, \ldots, \cdot)$ of $\mathbf{X}^{\star}$, which is a function of $k \cdot n$ real variables, is obtained via a triangular norm $(t$-norm $T)$, i.e.

$$
\zeta\left(x_{1}, \ldots, x_{k \cdot n}\right)=T\left(\zeta_{1}\left(x_{1}, \ldots, x_{k}\right), \zeta_{2}\left(x_{k+1}, \ldots, x_{2 k}\right), \ldots, \zeta_{n}\left(x_{(n-1) k+1}, \ldots, x_{k \cdot n}\right)\right) .
$$

Applying the minimum- $t$-norm, the vector-characterizing function $\zeta(\cdot, \ldots, \cdot)$ of $\mathbf{X}^{\star}$ is obtained:

$$
\zeta\left(x_{1}, \ldots, x_{k \cdot n}\right)=\min _{i=1(1) n)}\left\{\zeta_{i}\left(x_{(i-1) k+1}, x_{(i-1) k+2}, \ldots, x_{(i-1) k+k}\right)\right\} \quad \forall\left(x_{1}, \ldots, x_{k \cdot n}\right) \in \mathbb{R}^{k \cdot n}
$$

For example, let $k=2$, i.e. $\mathbf{x}_{i}^{\star}=\left(x_{1 \cdot i}, x_{2 \cdot i}\right)^{\star} \widehat{=} \zeta(\cdot, \cdot), i=1(1) n$ (i.e. $n$ observations), the combined fuzzy sample $\mathbf{X}^{\star}$ is obtained by:

$$
\mathbf{X}^{\star}=\left(x_{1}, x_{2}, \ldots, x_{1 \cdot n}, x_{2 \cdot n}\right)^{\star} \widehat{=} \zeta\left(x_{1}, x_{2}, \ldots, x_{1 \cdot n}, x_{2 \cdot n}\right) \text { where } \zeta: \mathbb{R}^{2 \cdot n} \rightarrow[0,1]
$$

In this case, the vector-characterizing function $\zeta(\cdot, \ldots, \cdot)$ of the combined fuzzy sample is obtained in the following way: 


$$
\begin{aligned}
\zeta\left(x_{1}, x_{2}, \ldots, x_{1 \cdot n}, x_{2 \cdot n}\right): & =\min _{i=1(1) n)}\left\{\zeta_{i}\left(x_{1 \cdot i}, x_{2 \cdot i}\right)\right\} \\
& =\min \left\{\zeta_{1}\left(x_{1}, x_{2}\right), \ldots, \zeta_{n}\left(x_{1 \cdot n}, x_{2 \cdot n}\right)\right\} \quad \forall\left(x_{1}, x_{2}, \ldots, x_{1 \cdot n}, x_{2 \cdot n}\right) \in \mathbb{R}^{2 \cdot n}
\end{aligned}
$$

Lemma 2. Through the combination of $n$ fuzzy observaions $\boldsymbol{x}_{i}^{\star}, i=1(1) n$ of a $k$-dimensional fuzzy quantity with vector-characterizing functions $\zeta_{i}(\cdot)$ by the minimum-t-norm, $n$ fuzzy $k$ dimensional vectors are combined into an $(k \cdot n)$-dimensional fuzzy vector with vector-characterizing function $\zeta(\cdot, \ldots, \cdot)$, for which the following property holds:

$$
C_{\delta}[\zeta(\cdot, \ldots, \cdot)]=\times_{i=1}^{n} C_{\delta}\left[\zeta_{i}(\cdot)\right] \quad \forall \delta \in(0,1]
$$

where $\zeta\left(x_{1}, \ldots, x_{k \cdot n}\right)=\min _{i=1(1) n)}\left\{\zeta_{1}\left(x_{1}, \ldots, x_{k}\right), \zeta_{2}\left(x_{k+1}, \ldots, x_{2 k}\right), \ldots, \zeta_{n}\left(x_{(n-1) k+1}, \ldots, x_{k \cdot n}\right)\right\}$

$\forall\left(x_{1}, \ldots, x_{k \cdot n}\right) \in \mathbb{R}^{k \cdot n}$

The $\delta$-cuts of the combined fuzzy vector $\boldsymbol{X}^{\star}$ are the Cartesian products of the $\delta$-cuts of the fuzzy vectors $\boldsymbol{x}_{i}^{\star}, i=1(1) n$.

Proof. $C_{\delta}[\zeta(\cdot, \ldots, \cdot)]=\left\{\mathbf{x} \in \mathbb{R}^{k \cdot n}: \zeta(\mathbf{x}) \geq \delta\right\}$ $=\left\{\mathbf{x}: \min \left\{\zeta_{1}\left(x_{1}, \ldots, x_{k}\right), \zeta_{2}\left(x_{k+1}, \ldots, x_{2 k}\right), \ldots, \zeta_{n}\left(x_{(n-1) k+1}, \ldots, x_{k \cdot n}\right)\right\} \geq \delta\right\}$,

by $\mathbf{x} \in \mathrm{X}_{i=1}^{n} C_{\delta}\left[\zeta_{i}(\cdot)\right] \quad \Longleftrightarrow \quad \zeta_{i}(\cdot) \geq \delta \quad \forall i=1(1) n \quad \Longleftrightarrow \quad \min _{i=1(1) n)}\left\{\zeta_{i}(\cdot)\right\} \geq \delta$, and it follows $C_{\delta}[\zeta(\cdot, \ldots, \cdot)]=\mathrm{X}_{i=1}^{n} C_{\delta}\left[\zeta_{i}(\cdot)\right]$.

\section{Statistical functions of fuzzy multivariate data}

Data analysis methods generally use functions $f\left(x_{1}, \ldots, x_{n}\right), f: \mathbb{R}^{n} \rightarrow \mathrm{S}$, where $\mathrm{S}$ is a suitable adopted space, i.e. statistics, to summarize and explain data. In case of non-precise data $x_{1}^{\star}, \ldots, x_{n}^{\star}$, the function becomes $f\left(x_{1}^{\star}, \ldots, x_{k}^{\star}\right)$. The values $y^{\star}=f\left(x_{1}^{\star}, \ldots, x_{n}^{\star}\right)$ are nonprecise by the propagation of imprecision (fuzziness). The membership function of $y^{\star}$ is obtained via the extension principle based on combined fuzzy samples and minimum $t$-norm (see Definition 2). Through the combined fuzzy sample $\mathbf{X}^{\star}$ (or the fuzzy vector $\mathbf{x}^{\star}$ ) with its vector-characterizing function $\zeta(\cdot, \ldots, \cdot)$, the generalization of statistics $f\left(x_{1}, \ldots, x_{n}\right)$ to handle fuzzy data $x_{1}^{\star}, \ldots, x_{n}^{\star}$ is possible:

Using the notation $\mathbf{x}^{\star}=\left(x_{1}, \ldots, x_{n}\right) \in \mathbb{R}^{n}$ and $\eta(\cdot)$ for the membership function of $y^{\star}$, the values $\eta(y), \forall y \in \mathrm{S}$, are given by

$\eta(y)=\left\{\begin{aligned} \sup \left\{\zeta\left(x_{1}, \ldots, x_{n}\right): f\left(x_{1}, \ldots, x_{n}\right)=y\right\} & \text { if } \exists\left(x_{1}, \ldots, x_{n}\right): f\left(x_{1}, \ldots, x_{n}\right)=y \\ 0 & \text { if } \nexists\left(x_{1}, \ldots, x_{n}\right): f\left(x_{1}, \ldots, x_{n}\right)=y\end{aligned}\right\}$.

For a fuzzy vector $\mathbf{x}^{\star}$ in $\mathbb{R}^{n}$ with vector-characterizing function $\zeta(\cdot, \ldots, \cdot)$, all $\delta$-cuts $C_{\delta}\left[\mathbf{x}^{\star}\right]$ are defined as simply connected and compact subsets of $\mathbb{R}^{n}$. For continuous functions, the following holds:

Let $x_{1}^{\star}, \ldots, x_{n}^{\star}$ be fuzzy intervals, i.e. all $\delta$-cuts of $x_{i}^{\star}$ are finite closed intervals, and $f: \mathbb{R}^{n} \rightarrow \mathbb{R}$ be a continuous function, then $y^{\star}=f\left(x_{1}^{\star}, \ldots, x_{n}^{\star}\right)$ is a fuzzy interval whose $\delta$-cuts are given by

$$
C_{\delta}\left[y^{\star}\right]=\left[\min _{\mathbf{x} \in C_{\delta}\left[\mathbf{x}^{\star}\right]} f(\mathbf{x}), \max _{\mathbf{x} \in C_{\delta}\left[\mathbf{x}^{\star}\right]} f(\mathbf{x})\right] \quad \forall \delta \in(0,1]
$$

Proof. Since $x_{i}^{\star}, i=1(1) n$, are fuzzy intervals, the $\delta$-cuts of the combined fuzzy vector $\mathbf{x}^{\star}$ are Cartesian products of compact intervals. The continuity of $f(\cdot)$ implies that $f\left(C_{\delta}\left[\mathbf{x}^{\star}\right]\right)$ is compact and simply connected, and, therefore, an interval. By the continuity of $f(\cdot)$, it follows that $f^{-1}(\{y\})$ is closed and, thus, $\sup _{\mathbf{x} \in C_{\delta}\left[\mathbf{x}^{\star}\right]} f(\mathbf{x})=\max _{\mathbf{x} \in C_{\delta}\left[\mathbf{x}^{\star}\right]} f(\mathbf{x})$. As a result, $C_{\delta}\left[\mathbf{x}^{\star}\right]$ is a closed interval. 
In multivariate case, $(n \cdot k)$-dimensional fuzzy vector ( $n$ samples of $k$ variables) with (combined) vector-characterizing function $\zeta(\cdot, \ldots, \cdot)$, the value $y^{\star \star}$ of the function $f: \mathbb{R}^{k \cdot n} \rightarrow \mathrm{S}$ is defined to have the following membership function:

$$
\eta(y)=\left\{\begin{aligned}
\sup \left\{\zeta\left(x_{1}, \ldots, x_{k \cdot n}\right): f\left(x_{1}, \ldots, x_{k \cdot n}\right)=y\right\} & \text { if } \exists\left(x_{1}, \ldots, x_{k \cdot n}\right): f\left(x_{1}, \ldots, x_{k \cdot n}\right)=y \\
0 & \text { if } \nexists\left(x_{1}, \ldots, x_{k \cdot n}\right): f\left(x_{1}, \ldots, x_{k \cdot n}\right)=y
\end{aligned}\right\}
$$

For a combined fuzzy vector $\mathbf{X}^{\star}$ in $\mathbb{R}^{k \cdot n}$ with vector-characterizing function $\zeta(\cdot, \ldots, \cdot)$, the value $y^{\star \star}$, where $\mathrm{S}=\mathbb{R}$, all of the $\delta$-cuts $C_{\delta}\left[\mathbf{X}^{\star}\right]$ are defined as simply connected and compact subsets of $\mathbb{R}^{k \cdot n}$. For continuous functions, the following holds:

$$
C_{\delta}\left[y^{\star \star}\right]=\left[\min _{\mathbf{X} \in C_{\delta}\left[\mathbf{X}^{\star}\right]} f(\mathbf{X}), \max _{\mathbf{X} \in C_{\delta}\left[\mathbf{X}^{\star}\right]} f(\mathbf{X})\right] \quad \forall \delta \in(0,1]
$$

Note: As defined in Lemma 2, the $\delta$-cuts of the combined fuzzy sample $\mathbf{X}^{\star}$ are the Cartesian products of the $\delta$-cuts of the fuzzy vectors $\mathbf{x}_{i}^{\star}, i=1(1) n$.

\section{Generalized point estimators}

The essential characteristics of a univariate distribution are the arithmetic mean, as a measure of location, and the variance, as a measure of dispersion. Similarly, considering a data set, the sample mean and sample variance of the sample are important summary measures. Generalized estimators for fuzzy univariate data are explained in Viertl (2006).

In multivariate case, the means and variances of the separate measurements are of interest. An essential aspect of multivariate analysis is the dependence between the different variables, i.e. the dependence between two variables may involve the covariance between them. In this case, the relevant parameters are in forms of vector and/or matrix, e.g. the mean vector, the variance-covariance matrix, and the correlation coefficient matrix.

For a sample of fuzzy vectors $\mathbf{x}_{i}^{\star}, i=1(1) n$ in $\mathbb{R}^{k}$ with vector-characterizing functions $\zeta_{i}(\cdot, \ldots, \cdot)$, a point estimate from a statistic (function) $\vartheta\left(\mathbf{x}_{1}^{\star}, \ldots, \mathbf{x}_{n}^{\star}\right)$ becomes a fuzzy element $\hat{\theta}^{\star}$ in parameter space $\Theta$. Hence, for a combined fuzzy vector $\mathbf{X}^{\star}$ in $\mathbb{R}^{k \cdot n}$ with vector-characterizing function $\zeta(\cdot, \ldots, \cdot)$, the generalized point estimator based on a standard estimator $\vartheta: \mathbb{R}^{k \cdot n} \rightarrow \Theta$ yields a fuzzy point estimate $\hat{\theta}^{\star}$ whose membership function $\eta(\cdot)$ is defined by its values as follows:

$\eta(\theta)=\left\{\begin{array}{cc}\sup \left\{\zeta\left(x_{1}, \ldots, x_{k \cdot n}\right): \vartheta\left(x_{1}, \ldots, x_{k \cdot n}\right)=\theta\right\} & \text { if } \exists\left(x_{1}, \ldots, x_{k \cdot n}\right): \vartheta\left(x_{1}, \ldots, x_{k \cdot n}\right)=\theta \\ 0 & \text { if } \nexists\left(x_{1}, \ldots, x_{k \cdot n}\right): \vartheta\left(x_{1}, \ldots, x_{k \cdot n}\right)=\theta\end{array}\right\}$

All $\delta$-cuts $C_{\delta}\left[\mathbf{X}^{\star}\right]$ are assumed to be simply connected and compact subsets of $\mathbb{R}^{k \cdot n}$. For continuous functions $\vartheta(\cdot, \ldots, \cdot)$ and one-dimensional parameter $\theta$, the following holds:

$$
\begin{aligned}
& C_{\delta}\left[\hat{\theta}^{\star}\right]=\left[\min _{\mathbf{X} \in C_{\delta}\left[\mathbf{X}^{\star}\right]} \vartheta(\mathbf{X}), \max _{\mathbf{X} \in C_{\delta}\left[\mathbf{X}^{\star}\right]} \vartheta(\mathbf{X})\right] \quad \forall \delta \in(0,1] \\
& \text { or symbolically } \hat{\theta}^{\star}=\vartheta\left(\mathbf{X}^{\star}\right)
\end{aligned}
$$

Proof. The $\delta$-cuts of the combined fuzzy sample $\mathbf{X}^{\star}$ are the Cartesian products of the $\delta$-cuts of the fuzzy vectors $\mathbf{x}_{i}^{\star}, i=1(1) n$ (see Lemma 2 ). The continuity of $\vartheta(\cdot)$ implies that $\vartheta\left(C_{\delta}\left[\mathbf{X}^{\star}\right]\right.$ ) is compact and simply connected, therefore, an interval. By the continuity of $\vartheta(\cdot)$, it also follows that $\vartheta^{-1}(\{\hat{\theta}\})$ is closed for all $\hat{\theta} \in \Theta$ and, thus, $\sup _{\mathbf{X} \in C_{\delta}\left[\mathbf{X}^{\star}\right]} \vartheta(\mathbf{X})=\max _{\mathbf{X} \in C_{\delta}\left[\mathbf{X}^{\star}\right]} \vartheta(\mathbf{X})$. As a result, $C_{\delta}\left[\mathbf{X}^{\star}\right]$ is a closed interval.

\subsection{Point estimator for mean vector}

The arithmetic mean is one of the important parameters that characterize the distribution. In multivariate analysis, the mean vector $\boldsymbol{\mu}$ is of interest, where 


$$
\boldsymbol{\mu}=\left[\begin{array}{c}
\mu_{1} \\
\mu_{2} \\
\vdots \\
\mu_{k}
\end{array}\right] .
$$

Let $\mathbf{X}^{\star}$ be a combined fuzzy vector in $\mathbb{R}^{k \cdot n}$ with vector-characterizing function $\zeta(\cdot, \ldots, \cdot)$, the $\delta$-cut $C_{\delta}\left[\mathbf{X}^{\star}\right]$ is defined as simply connected and compact subset of $\mathbb{R}^{k \cdot n}$.

For continuous functions $f: \mathbb{R}^{k \cdot n} \rightarrow \mathbb{R}$ with fuzzy point estimate $\hat{\mu}_{j}^{\star}$ for the mean, the following holds:

$$
C_{\delta}\left[\hat{\mu}_{j}^{\star}\right]=\left[\min _{\mathbf{X} \in C_{\delta}\left[\mathbf{X}^{\star}\right]} f_{\mu_{j}}(\mathbf{X}), \max _{\mathbf{X} \in C_{\delta}\left[\mathbf{X}^{\star}\right]} f_{\mu_{j}}(\mathbf{X})\right] \quad \forall \delta \in(0,1] .
$$

Remark 1. Minimum and maximum of $f_{\mu_{j}}(\mathbf{X})$ are defined as: $\min f_{\mu_{j}}(\mathbf{X})=f_{\mu_{j}}(\underline{\mathbf{X}})=$ $\frac{1}{n} \sum_{i=1}^{n} \underline{x}_{i j, \delta}$ and $\max f_{\mu_{j}}(\mathbf{X})=f_{\mu_{j}}(\overline{\mathbf{X}})=\frac{1}{n} \sum_{i=1}^{n} \bar{x}_{i j, \delta}$, where $\underline{x}_{i j, \delta}$ and $\bar{x}_{i j, \delta}$ are the lower and upper limits of the $\delta$-cut of a fuzzy interval at a specified $\delta$-level, respectively.

Accordingly, the membership function $\eta(\cdot)$ (as generalized in Equation (5)) of the fuzzy point estimate $\hat{\mu}_{j}^{\star}$ can be constructed numerically through lower and upper limits of multiple $\delta$-cuts as well.

\subsection{Point estimator for variance-covariance matrix}

The sample variance measures how far a set of numbers is spread out. Mathematically, it is simply the average of the squares of the deviations of observations about their sample mean. On the other hand, sample covariance is defined in terms of cross products between two variables (factors) of interest. The sample variance-covariance matrix is an unbiased estimator of the covariance matrix $\boldsymbol{\Sigma}$. For a standard sample $\mathbf{x}^{\star}=\left(x_{1}, \ldots, x_{k \cdot n}\right)$, each $i j$ element in the matrix of sums of squares and cross products of deviations about the sample mean $\overline{\mathbf{x}}$ is generally defined as:

$$
\mathbf{A}_{i \times j}: a_{i j}=\sum_{i=1}^{n}\left(x_{i j}-\overline{\mathbf{x}}\right)\left(x_{i j}-\overline{\mathbf{x}}\right)^{\prime} \text {; where } i=1(1) n, j=1(1) k \text {, and } n>k \text {. }
$$

Remark 2. The constraint $n>k$ is necessary, so that $\mathbf{A}$ is positive definite (Anderson 2003).

The standard point estimator of the variance $\sigma_{i}^{2}$ is

$\hat{\sigma}_{i}^{2}=(1 /(n-1)) \cdot a_{i j}=(1 /(n-1)) \sum_{i=1}^{n}\left(x_{i j}-\overline{\mathbf{x}}_{j}\right)\left(x_{i j}-\overline{\mathbf{x}}_{j}\right)^{\prime}=(1 /(n-1))\left(\sum_{i=1}^{n} x_{i j}^{2}-n \bar{x}_{j}^{2}\right)$.

Hence, the estimated variance-covariance matrix is derived as:

$$
\widehat{\Sigma}_{l \times m}: \hat{\sigma}_{l m}=(1 /(n-1)) \sum_{i=1}^{n}\left(x_{i l}-\bar{x}_{l}\right)\left(x_{i m}-\bar{x}_{m}\right) ; l=1(1) k, m=1(1) k .
$$

Let $\mathbf{X}^{\star}$ be a combined fuzzy vector in $\mathbb{R}^{k \cdot n}$ with vector-characterizing function $\zeta(\cdot, \ldots, \cdot)$, $\delta$-cut $C_{\delta}\left[\mathbf{X}^{\star}\right]$ is defined as simply connected and compact subset of $\mathbb{R}^{k \cdot n}$. For continuous standard estimation $f: \mathbb{R}^{k \cdot n} \rightarrow \mathbb{R}$ and the corresponding fuzzy point estimate $\hat{\sigma}_{l m}^{\star}$ for the variance-covariance, the following holds:

$$
C_{\delta}\left[\hat{\sigma}_{l m}^{\star}\right]=\left[\min _{\mathbf{X} \in C_{\delta}\left[\mathbf{X}^{\star}\right]} f_{\sigma_{l m}}(\mathbf{X}), \max _{\mathbf{X} \in C_{\delta}\left[\mathbf{X}^{\star}\right]} f_{\sigma_{l m}}(\mathbf{X})\right] \quad \forall \delta \in(0,1] .
$$

For sample variance-covariance matrix, minimum and maximum of $f_{\sigma_{l m}}(\mathbf{X})$ are defined as: $\min f_{\sigma_{l m}}(\mathbf{X})=\min \hat{\sigma}_{l m}\left(\mathbf{x}_{\min }\right)$ and $\max f_{\sigma_{l m}}(\mathbf{X})=\max \hat{\sigma}_{l m}\left(\mathbf{x}_{\max }\right)$

where $\mathbf{x}_{\min }$ and $\mathbf{x}_{\max }$ vectors contain the observations that lie closest to and farthest from the corresponding sample means, hence, resulted in minimum and maximum sample variances (covariances) at the specified $\delta$-level respectively.

\subsection{Point estimator for correlation matrix}

It is frequently informative to separate the information contained in variances from that con- 
tained in measures of association and, in particular, the measure of association known as the population correlation coefficient $\left(\rho_{l m}\right)$ Wichern and Johnson (2007). Symbolically, the estimated fuzzy sample correlation coefficients are obtained from the following estimator (11), in a similar manner as the standard sample correlation coefficient $\left(r_{l m}\right)$, but in forms of $\min r_{l m}$ and $\max r_{l m}$, where $l$ and $m$ represent two distinct variables of interest:

$$
f_{\rho_{l m}}(\mathbf{X})=r_{l m}=\frac{\sum_{i=1}^{n}\left(x_{i l}-\bar{x}_{l}\right)\left(x_{i m}-\bar{x}_{m}\right)}{\sqrt{\sum_{i=1}^{n}\left(x_{i l}-\bar{x}_{l}\right)^{2}} \sqrt{\sum_{i=1}^{n}\left(x_{i m}-\bar{x}_{m}\right)^{2}}}
$$

Applying the $\delta$-cuts $C_{\delta}\left[\mathbf{X}^{\star}\right]$, the lower and upper boundaries of the estimated sample correlation coefficients are obtained through the following simple linear programs (Liu and Kao 2002):

$$
\begin{gathered}
\min r_{l m, \delta}=\left\{\begin{array}{c}
\min \frac{\sum_{i=1}^{n}\left(x_{i l, \delta}-\bar{x}_{l, \delta}\right)\left(x_{i m, \delta}-\bar{x}_{m, \delta}\right)}{\sqrt{\sum_{i=1}^{n}\left(x_{i l, \delta}-\bar{x}_{l, \delta}\right)^{2}} \sqrt{\sum_{i=1}^{n}\left(x_{i m, \delta}-\bar{x}_{m, \delta}\right)^{2}}} \\
\text { s.t. } \underline{x}_{i l, \delta} \leq x_{i l, \delta} \leq \bar{x}_{i l, \delta} \quad \forall l \\
\underline{x}_{i m, \delta} \leq x_{i m, \delta} \leq \bar{x}_{i m, \delta} \quad \forall m \\
l=1(1) k, m=1(1) k
\end{array}\right\} \\
\max r_{l m, \delta}=\left\{\begin{array}{c}
\max \frac{\sum_{i=1}^{n}\left(x_{i l, \delta}-\bar{x}_{l, \delta}\right)\left(x_{i m, \delta}-\bar{x}_{m, \delta}\right)}{\sqrt{\sum_{i=1}^{n}\left(x_{i l, \delta}-\bar{x}_{l, \delta}\right)^{2}} \sqrt{\sum_{i=1}^{n}\left(x_{i m, \delta}-\bar{x}_{m, \delta}\right)^{2}}} \\
\text { s.t. } \underline{x}_{i l, \delta} \leq x_{i l, \delta} \leq \bar{x}_{i l, \delta} \quad \forall l \\
\underline{x}_{i m, \delta} \leq x_{i m, \delta} \leq \bar{x}_{i m, \delta} \quad \forall m \\
l=1(1) k, m=1(1) k
\end{array}\right\}
\end{gathered}
$$

For continuous functions $f: \mathbb{R}^{k \cdot n} \rightarrow \mathbb{R}$ with fuzzy point estimate $\hat{\rho}_{l m}^{\star}$ for the correlation coefficient, where $l$ and $m$ represent two distinct variables of interest, the following holds respectively:

$$
C_{\delta}\left[\hat{\rho}_{l m}^{\star}\right]=\left[\min _{\mathbf{X} \in C_{\delta}\left[\mathbf{X}^{\star}\right]} f_{\rho_{l m}}(\mathbf{X}), \max _{\mathbf{X} \in C_{\delta}\left[\mathbf{X}^{\star}\right]} f_{\rho_{l m}}(\mathbf{X})\right] \quad \forall \delta \in(0,1]
$$

For sample correlation matrix, minimum and maximum of $f_{\rho_{l m}}(\mathbf{X})$ are defined as: $\min f_{\rho_{l m}}(\mathbf{X})=$ $\min r_{l m}$ and $\max f_{\rho_{l m}}(\mathbf{X})=\max r_{l m}$. Through the lower and upper boundaries of multiple $\delta$ cuts, i.e. $\min r_{l m, \delta}$ and $\max r_{l m, \delta}$, the membership function $\eta_{l m}(\cdot)$ of the fuzzy point estimate $\hat{\rho}_{l m}^{\star}$ can be obtained numerically.

Example. As a simplified example, a part of the results of a full-factorial experiment is adapted. Consider results of flat lapping measurements of stainless steel specimens (Table 1), where the process parameters of interest are obtained surface roughness, measured by mean of arithmetic mean $\mathrm{Ra}\left(\mathrm{x} 10^{3} \mathrm{~mm}\right) x_{1}^{\star}$, and calculated material removal rate MRR $\left(\mathrm{x} 10^{-3}\right.$ $\mathrm{mm} / \mathrm{s}) x_{2}^{\star}$. These measurements are naturally uncertain, i.e. considered as fuzzy numbers $x_{n}^{\star}=[a, b, c, d]_{n}$ (intuitively with trapezoidal-shape membership functions) and consequently they are further together considered as vector of fuzzy number $\mathbf{x}_{i}^{\star}=\left[x_{1}^{\star}, x_{2}^{\star}\right]$ where $i=1,2, \ldots, 8$ (two variables of eight observations).

Table 1: Results of flat lapping measurements

\begin{tabular}{|c|c|c|c|c|c|c|c|c|}
\hline Expt. & \multicolumn{3}{|c|}{ Ra (1000th-mm) } & \multicolumn{4}{c|}{ MRR (1000th-mm/s) } \\
\cline { 2 - 9 } No. & $\mathrm{a}$ & $\mathrm{b}$ & $\mathrm{c}$ & $\mathrm{d}$ & $\mathrm{a}$ & $\mathrm{b}$ & $\mathrm{c}$ & $\mathrm{d}$ \\
\hline 1 & 0.001 & 0.051 & 0.101 & 0.151 & 0.022 & 0.037 & 0.057 & 0.077 \\
2 & 0.021 & 0.071 & 0.121 & 0.171 & 0.176 & 0.191 & 0.211 & 0.231 \\
3 & 0.025 & 0.075 & 0.125 & 0.125 & 0.008 & 0.025 & 0.043 & 0.065 \\
4 & 0.032 & 0.082 & 0.132 & 0.182 & 0.005 & 0.020 & 0.040 & 0.060 \\
5 & 0.038 & 0.088 & 0.132 & 0.188 & 0.001 & 0.016 & 0.036 & 0.056 \\
6 & 0.045 & 0.095 & 0.145 & 0.195 & 0.013 & 0.028 & 0.048 & 0.068 \\
7 & 0.052 & 0.102 & 0.152 & 0.202 & 0.167 & 0.182 & 0.202 & 0.222 \\
8 & 0.058 & 0.108 & 0.158 & 0.208 & 0.026 & 0.041 & 0.061 & 0.081 \\
\hline
\end{tabular}


Through the method of combining fuzzy samples described in Section 3, the corresponding combined fuzzy vector $\mathbf{X}^{\star}=\left(x_{1 \cdot i}, x_{2 \cdot i}\right)^{\star}$ is obtained along with its vector-characterizing function $\zeta\left(x_{1}, x_{2}, \ldots, x_{1 \cdot 8}, x_{2 \cdot 8}\right)$. As defined in equation $(7)$, the fuzzy point estimate $\hat{\mu}_{j}^{\star}$ for the mean can be determined as lower and upper boundaries of the estimated sample mean at different $k \delta$-levels $\left[\overline{\mathbf{X}}_{\delta_{k}}^{L}, \overline{\mathbf{X}}_{\delta_{k}}^{U}\right]$ through $\overline{\mathbf{X}}_{\delta_{k}}^{L}=\min f_{\mu_{j}}(\mathbf{X})=f_{\mu_{j}}(\underline{\mathbf{X}})=\frac{1}{n} \sum_{i=1}^{n} \underline{x}_{i j, \delta_{k}}$ and $\overline{\mathbf{X}}_{\delta_{k}}^{U}=\max f_{\mu_{j}}(\mathbf{X})=f_{\mu_{j}}(\overline{\mathbf{X}})=\frac{1}{n} \sum_{i=1}^{n} \bar{x}_{i j, \delta_{k}}$ :

$$
\begin{aligned}
& \overline{\mathbf{X}}_{\delta=0+}=\left[\begin{array}{l}
{[0.034,0.178]} \\
{[0.052,0.107]}
\end{array}\right] \\
& \overline{\mathbf{X}}_{\delta=0.25}=\left[\begin{array}{l}
{[0.046,0.167]} \\
{[0.052,0.102]}
\end{array}\right] \\
& \overline{\mathbf{X}}_{\delta=0.50}=\left[\begin{array}{l}
{[0.059,0.156]} \\
{[0.060,0.097]}
\end{array}\right] \\
& \overline{\mathbf{X}}_{\delta=0.75}=\left[\begin{array}{l}
{[0.071,0.145]} \\
{[0.063,0.092]}
\end{array}\right] \\
& \overline{\mathbf{X}}_{\delta=1}=\left[\begin{array}{l}
{[0.084,0.134]} \\
{[0.067,0.087]}
\end{array}\right]
\end{aligned}
$$

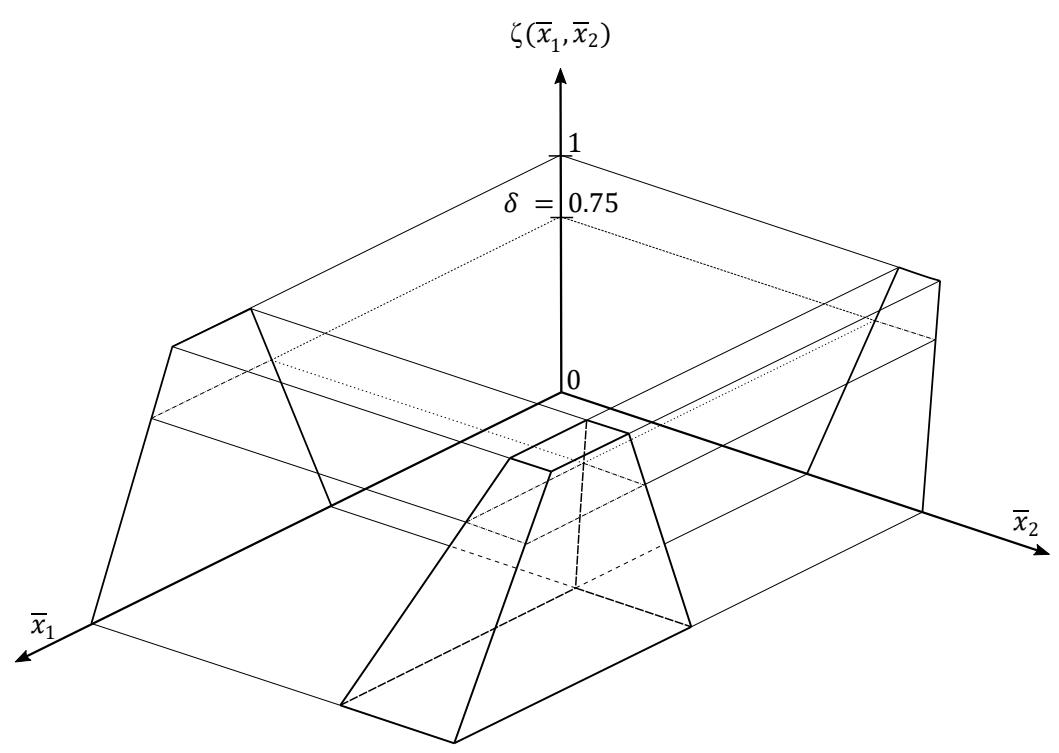

Figure 1: Vector-characterizing function $\zeta\left(\bar{x}_{1}, \bar{x}_{2}\right)$ of the mean of combined fuzzy vector $\mathbf{X}^{\star}$

Note: At each $\delta$-level, a plane of rectangle is formed, e.g. $\overline{\mathbf{X}}_{\delta=0.75}=[0.071,0.145]$ x $[0.063,0.092]$.

As shown in Figure 1, vector-characterizing function of the mean of the combined fuzzy vector $\mathbf{X}^{\star}$ is of a polygon form. For each $\delta$-cut, a plane representing feasible mean values is obtained as a result.

Next, the search for the minimum and maximum of the variance-covariance continues, i.e. through the feasible regions that are closest/farthest from the means and those with resulting minimum/maximum cross products for the variance-covariance matrix. As defined in Equation (10), the lower and upper boundaries of the $\delta$-cut of the corresponding sample point 
estimate for fuzzy variance-covariance at different $\delta$-levels are

$$
\begin{aligned}
& \min \widehat{\boldsymbol{\Sigma}}_{\delta_{k}} \quad \max \widehat{\boldsymbol{\Sigma}}_{\delta_{k}} \\
& \widehat{\boldsymbol{\Sigma}}_{\delta=0+}=\left[\begin{array}{lr}
0.00034 & -0.00018 \\
& 0.00144
\end{array}\right]\left[\begin{array}{ll}
0.03095 & 0.00009 \\
& 0.01641
\end{array}\right] \\
& \widehat{\mathbf{\Sigma}}_{\delta=0.25}=\left[\begin{array}{ll}
0.00034 & 0.00003 \\
& 0.00181
\end{array}\right]\left[\begin{array}{ll}
0.02228 & 0.00009 \\
& 0.01316
\end{array}\right] \\
& \widehat{\mathbf{\Sigma}}_{\delta=0.50}=\left[\begin{array}{lr}
0.00034 & 0.00004 \\
\widehat{\mathbf{\Sigma}}_{\delta=0.75} & 0.00198
\end{array}\right]\left[\begin{array}{lr}
0.00034 & 0.00005 \\
& 0.00251
\end{array}\right]\left[\begin{array}{ll}
0.00922 & 0.00009 \\
& 0.01034
\end{array}\right] \\
& \widehat{\mathbf{\Sigma}}_{\delta=1}=\left[\begin{array}{ll}
0.00022 & -0.00001 \\
& 0.00321
\end{array}\right]\left[\begin{array}{ll}
0.00483 & 0.00009 \\
& 0.00866
\end{array}\right] .
\end{aligned}
$$

Note: Since both combined sample itself and the mean are fuzzy, with respect to the shapes of their vector-characterizing functions, for different $\delta$-cuts, the distances between the boundaries of the fuzzy sample and those of the fuzzy mean remain the same, i.e. the boundaries (limits) of the functions move in the same direction with the same distance. Hence, the same value of the $\mathrm{min} / \max$ variances at different $\delta$-levels of $x_{1}^{\star}$ and $x_{2}^{\star}$ respectively.

Finally, after applying the $\delta$-cuts as defined in Equation (14), the lower and upper boundaries of the estimated sample correlations $\min r_{l m, \delta}$ and $\max r_{l m, \delta}$ are obtained:

$$
\begin{aligned}
& \min r_{l m, \delta} \quad \max r_{l m, \delta} \\
& r_{l m, \delta=0+}=\left[\begin{array}{cc}
1 & -0.00784 \\
1
\end{array}\right]\left[\begin{array}{cc}
1 & 0.16462 \\
1 & 1
\end{array}\right] \\
& r_{l m, \delta=0.25}=\left[\begin{array}{cc}
1 & 0.00152 \\
1
\end{array}\right]\left[\begin{array}{cc}
1 & 0.11284 \\
1
\end{array}\right] \\
& r_{l m, \delta=0.50}=\left[\begin{array}{cc}
1 & 0.00277 \\
1
\end{array}\right]\left[\begin{array}{cc}
1 & 0.10828 \\
1
\end{array}\right] \\
& r_{l m, \delta=0.75}=\left[\begin{array}{cc}
1 & 0.00502 \\
1
\end{array}\right]\left[\begin{array}{cc}
1 & 0.09651 \\
1
\end{array}\right] \\
& r_{l m, \delta=0.1}=\left[\begin{array}{cc}
1 & -0.00168 \\
1 & 0.10530 \\
1 & 1
\end{array}\right]
\end{aligned}
$$

The resulting vector-characterizing function is also of a complex form due to fuzzy multiplication and division. As opposed to the maximum covariances, the different in maximum correlations are a result of different minimums of variance products. However, the quantitative values of covariance and correlation follow the same trend in the end. As indicated by the numerical values of correlation coefficient, based on the experimental results, the linear relationship between obtained surface roughness and material removal rate is trivial. In lapping theory, there are many factors that influence the quality of the obtained surface. In other words, a combination of factors, e.g. surface flatness, surface area, abrasive compound, lapping mechanism through rolling, scraping, and embedding, are needed to be considered altogether. 


\section{Final remarks}

The standard statistical inference methods are extended for multivariate fuzzy data. In particular, the concepts of fuzzy vector and fuzzy multivariate data are described. The method of combining $n$ observations of $k$-dimension is defined and served as focal point of the subsequent definitions. As a result, the combined fuzzy sample along with its corresponding vector-characterizing function is obtained. Accordingly, the generalized methods are then defined for the estimators of sample mean, variance-covariance, and correlation coefficient matrices for multivariate fuzzy data. A numerical example of a two-variable case through $\delta$-cut calculation is also provided. Because of increasing cardinality of the resulting Cartesian products, the higher the dimensions of considering variables are, the more complex the analysis it is. In other words, nonlinearity and nonconvexity may be expected from multivariate analysis. Hence, the results and interpretation depend rather on the adopted numerical methods and the data themselves as well. The proposed generalized method for multivariate fuzzy data provides a succinct alternative of mathematical definitions for the estimation of different statistics and can be further extended to the generalization of estimation of confidence regions, statistical tests, and Bayes' theorem in a natural way.

\section{References}

Anderson TW (2003). An Introduction to Multivariate Statistical Analysis. Wiley, NJ.

Blanco-Fernàndez A, et al. (2014). "A Distance-based Statistical Analysis of Fuzzy Numbervalued Data." International Journal of Approximate Reasoning, 55, 1487-1501.

Bustince H, Burillo P (1995). "Correlation of Interval-valued Intuitionistic Fuzzy Sets." Fuzzy Sets and Systems, 74, 237-244.

Dubois D, Prade H (1987). "The Mean Value of a Fuzzy Number." Fuzzy Sets and Systems, 24, 279-300.

Frühwirth-Schnatter S (1992). "Statistical Inference for Fuzzy Data." Fuzzy Sets and Systems, 50, 143-165.

Klir G, Yuan B (1995). Fuzzy Sets and Fuzzy Logic - Theory and Applications. Wiley, NJ.

Körner R (1997). "On the Variance of Fuzzy Random Variables." Fuzzy Sets and Systems, 92, 83-93.

Kruse R (1984). "Statistical Estimation with Linguistic Data." Information Science, 33, $197-207$.

Kruse R (1987). "On the Variance of Random Sets." Journal of Math. Analysis and Applications, 122, 469-473.

Kruse R, Meyer KD (1987). Statistics with Vague Data. D. Reidel Publishing, Dordrecht.

Liu ST, Kao C (2002). "Fuzzy Measures for Correlation Coefficient of Fuzzy Numbers." Fuzzy Sets and Systems, 128, 267-275.

Nguyen HT, Wu B (2006). "Random and Fuzzy Sets in Coarse Data Analysis." Computational Statistics and Data Analysis, 51(1), 70-85.

Ni Y, Cheung JY (2003). "Correlation Coefficient Estimate for Fuzzy Data." Intelligent Systems Design and Applications: Advances in Soft Computing, 23, 105-114.

Saneifard R, Saneifard R (2012). "Correlation Coefficient between Fuzzy Numbers Based on Central Interval." Journal of Fuzzy Set Valued Analysis, 2012, 1-9. 
Viertl R (2006). "Univariate Statistical Analysis with Fuzzy Data." Journal of Computational Statistics and Data Analysis, 51(1), 133-147.

Viertl R (2011). Statistical Methods for Fuzzy Data. Wiley, Chichester.

Viertl R (2015). "Measurement of Continuous Quantities and Their Statistical Evaluation." Austrian Journal of Statistics, 44, 25-32.

Viertl R, Hareter D (2006). Beschreibung und Analyse unscharfer Information. Springer, Wien.

Viertl R, Sunanta O (2013). "Fuzzy Bayesian Inference." METRON, 71(3), 207-216.

Voxman W (1998). "Some Remarks on Distances between Fuzzy Numbers." Fuzzy Sets and Systems, 100, 353-365.

Wang D (2004). "A Note on Consistency and Unbiasedness of Point Estimation with Fuzzy Data." Metrika, 60, 93-104.

Wichern DW, Johnson RA (2007). Applied Multivariate Statistical Analysis. Pearson Prentice Hall, NJ.

Zadeh LA (1965). "Fuzzy Sets." Information and Control, 8(1), 338-353.

Zadeh LA (1975). "The Concept of Linguistic Variable and Its Application to Approximate Reasoning." Information Science, 8, 43-80.

\section{Affiliation:}

Owat Sunanta

Institute of Statistics and Mathematical Methods in Economics

Vienna University of Technology

A-1040 Vienna, Austria

Telephone: $+43 / 588 / 011-0584$

E-mail: owat.sunanta@tuwien.ac.at

\section{Austrian Journal of Statistics}

published by the Austrian Society of Statistics

Volume 47

February 2018 http://www .ajs.or.at/

http://www.osg.or.at/

Submitted: 2016-03-18

Accepted: 2017-08-09 